\title{
GUSELKUMAB IN PSORIASIS PATIENTS WITH A HISTORY OF MALIGNANCY: 5-YEAR SAFETY FROM VOYAGE $1 \& 2$
}

Andrew Blauvelt, ${ }^{1}$ Diamant Thaçi, ${ }^{2}$ Kim A Papp, ${ }^{3}$ Vincent Ho, ${ }^{4}$ Kamran Ghoreschi, ${ }^{5}$ Byung Soo Kim, ${ }^{6}$ Megan Miller, ${ }^{7}$ Yaung-Kaung Shen, ${ }^{7}$ Yin You, ${ }^{7}$ Jenny Yu, ${ }^{7}$ Ya-Wen Yang, ${ }^{8}$ Jeff Crowley, ${ }^{9}$ Peter Foley ${ }^{10}$

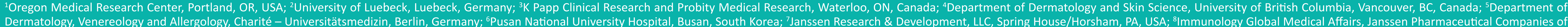
Dermatology, Venereology and Allergology, Charité - Universitätsmedizin, Berlin, Germany; ${ }^{6}$ Pusan National University Hospital, Busan, South Korea; ’ Janssen Research \& Development, LLC, Spring House/Horsham, PA, USA; 8 Immunology Global Medical Affairs,

BACKGROUND/OBJECTIVE

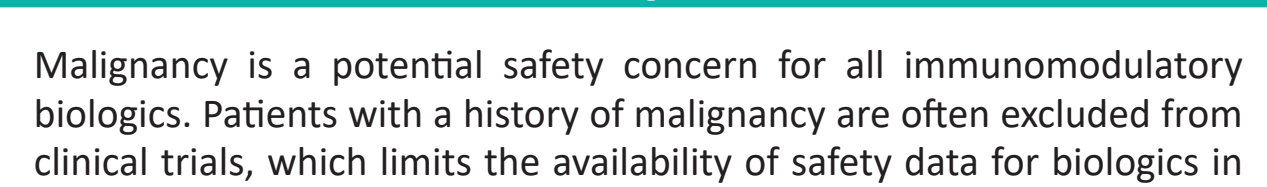
Clinical trials, which limits the availability of safety datat for biologics in
this population

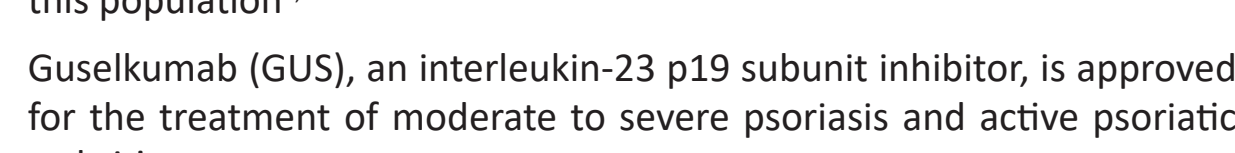

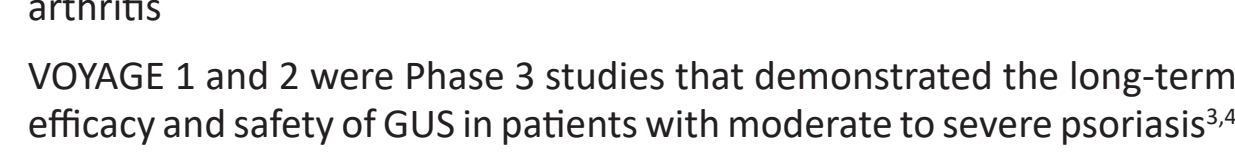
These studies included a small number of patients with a history of
malignancy (excluding non-melanoma skin cancer [NMSc]) at baseline

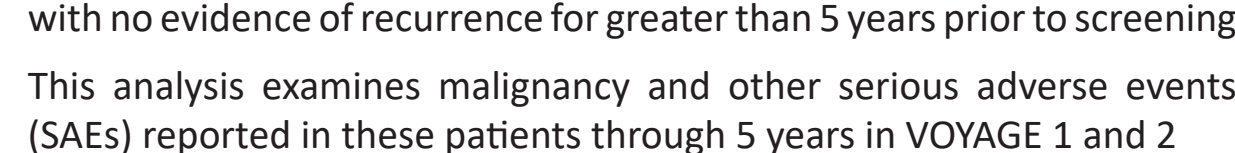

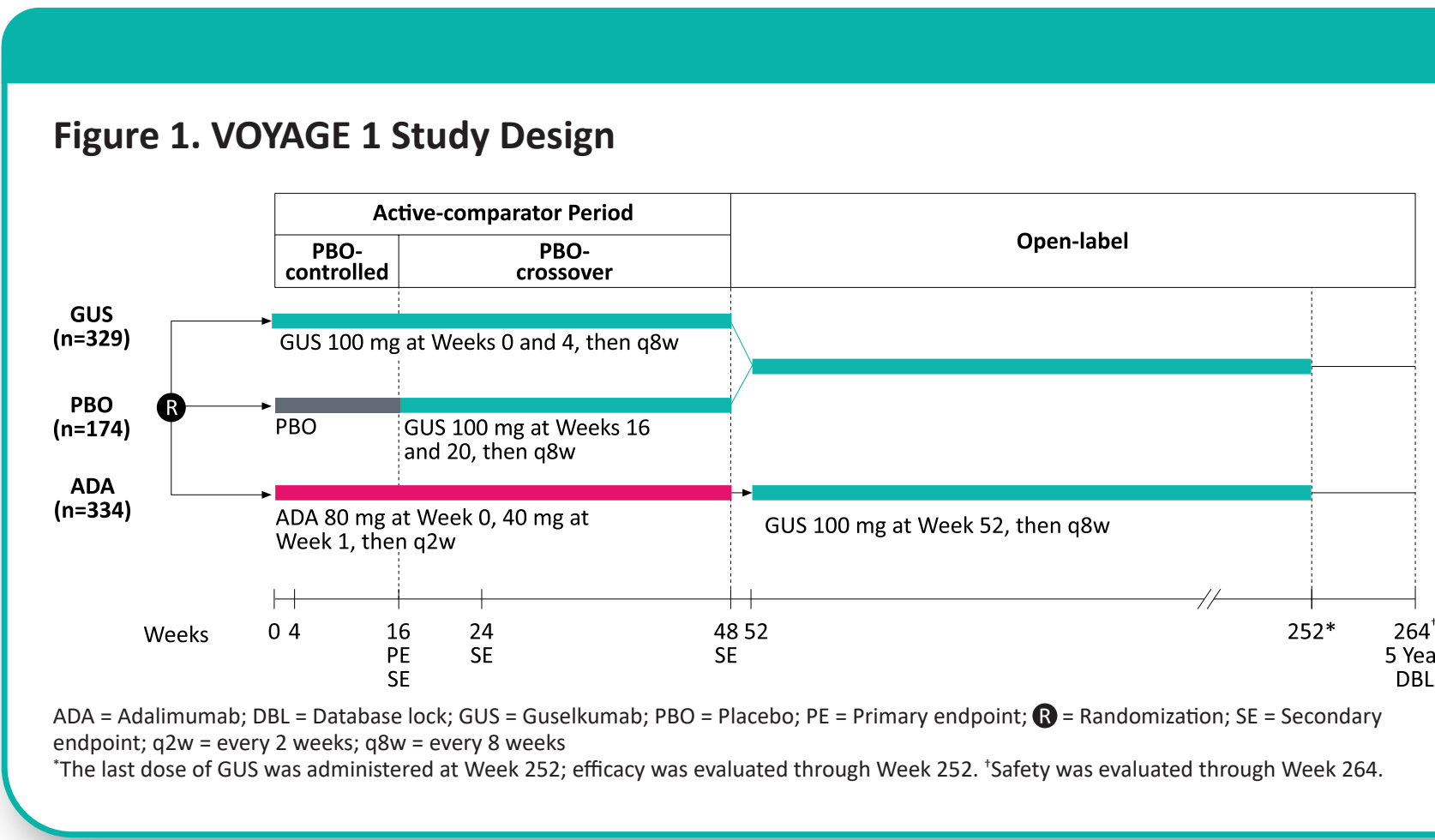

METHODS

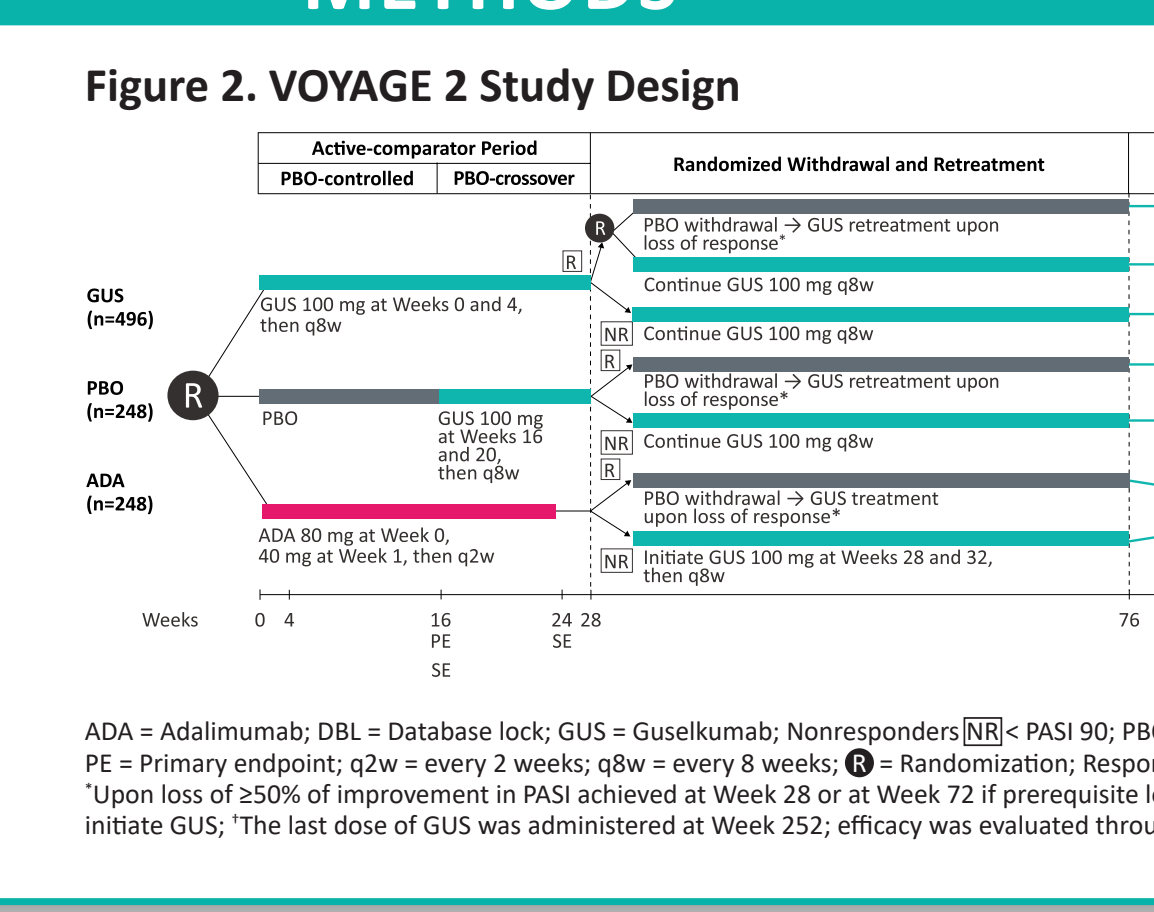

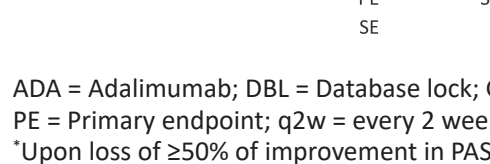

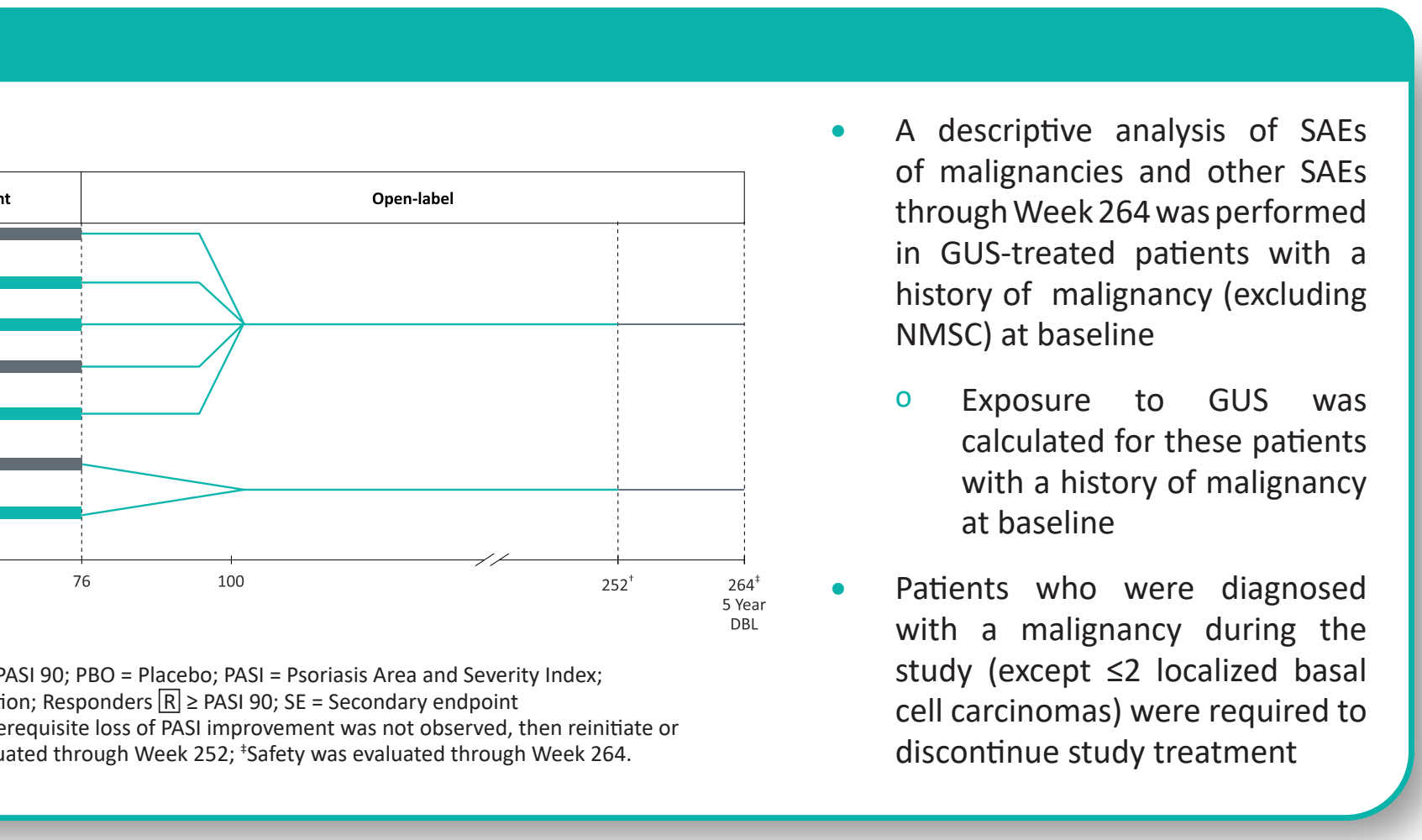

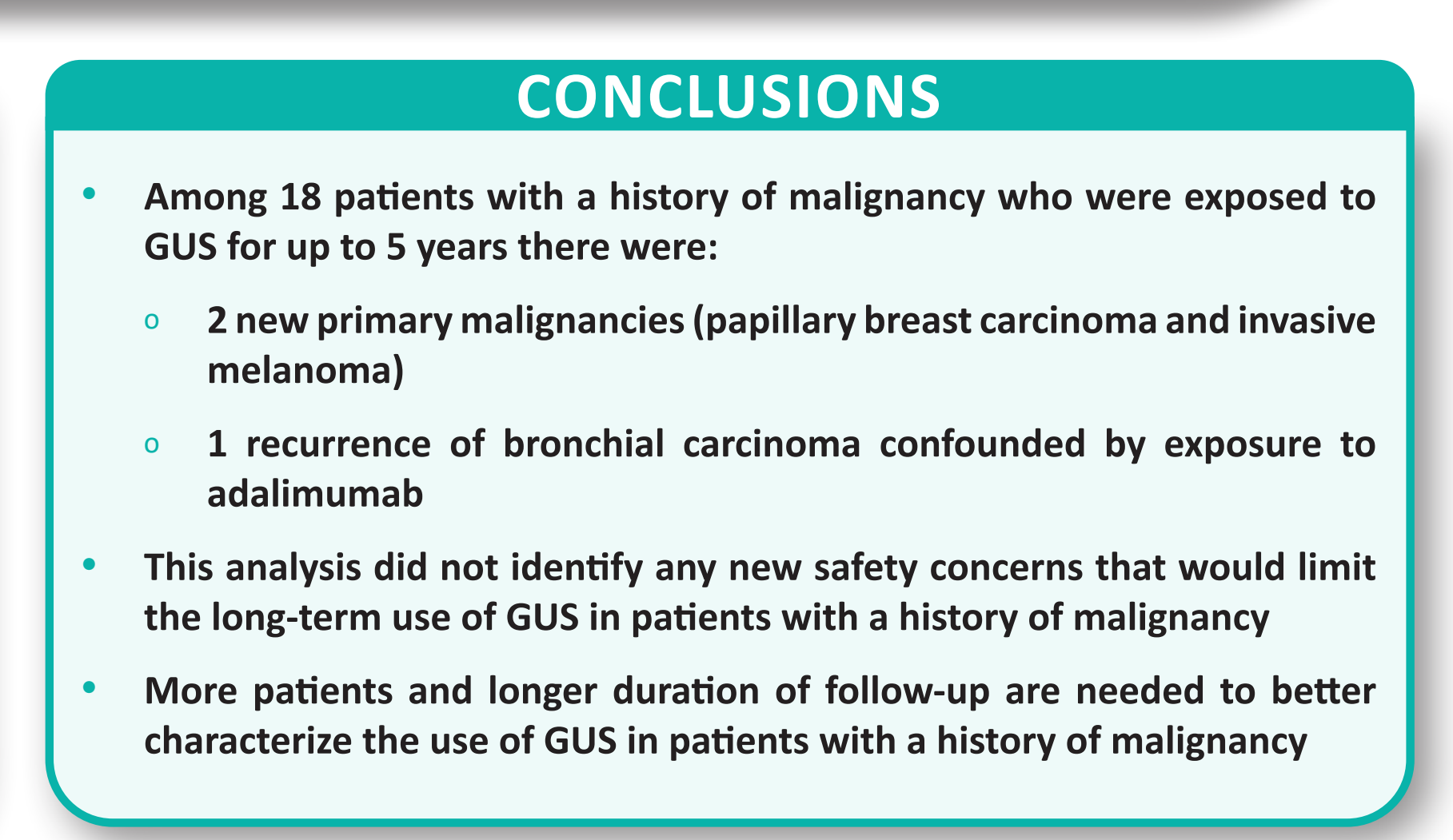

Patients With a History of Malignancy at Baseline

Of 1721 GUS. treated patients, $18(1.0 \%)$ had a prior history of malignancy at baseline

Mean (SD) exposure to GUS during the VOYAGE 1 or 2 study=184 (87) weeks; median (range)=225.5 (20-254) weeks

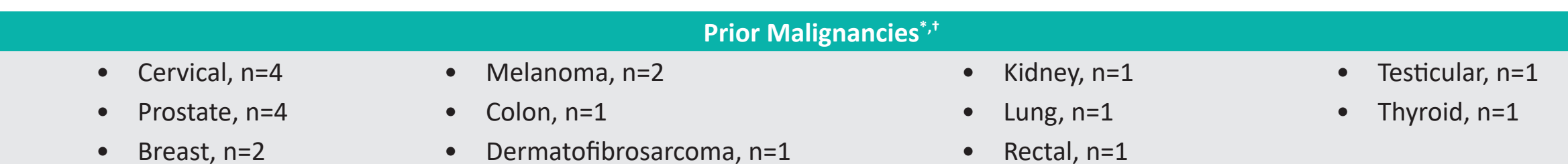

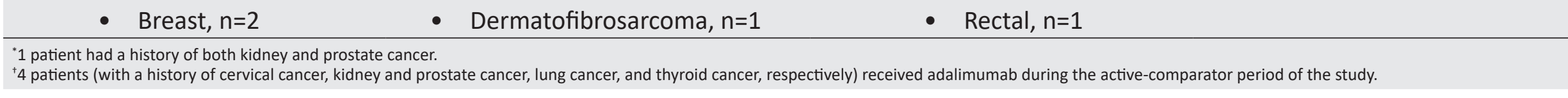

Of these 18 patients, 3 experienced SAEs of malignancy while participating in VOYAGE 1 or 2 for up to 5 years

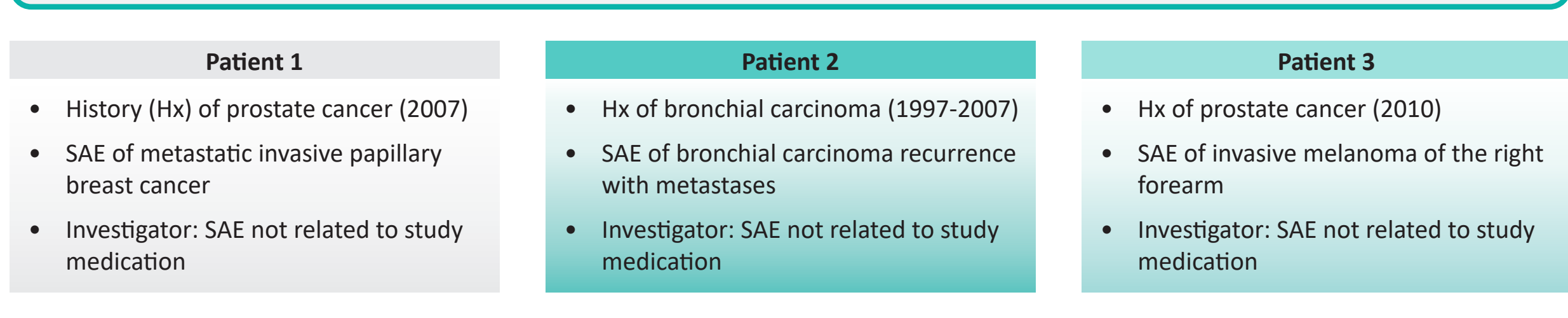

Patient 1: SAE of Metastatic Breast Cancer

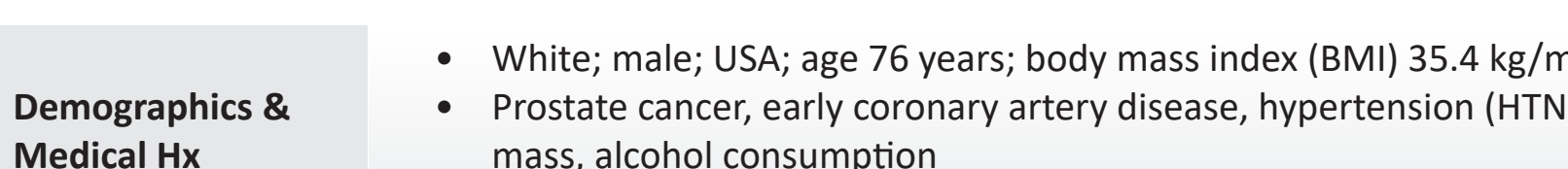

$$
\begin{aligned}
& \text { Prostate cancer, early coronary artery disease, hype } \\
& \text { mams alcohol consumption } \\
& \text { Prior psoralen with ultraviolet A, topical treatment }
\end{aligned}
$$

VOYAGE 1
Treatment

: Randomized to Gus

: Right breast lump observed $\sim 1$ year prior to study entry
Lump stowly enlarged and became tender

Diagnosis on Day 202:2.7.7 cm subareolar right breast mass
Gene mutation identification testing was negative

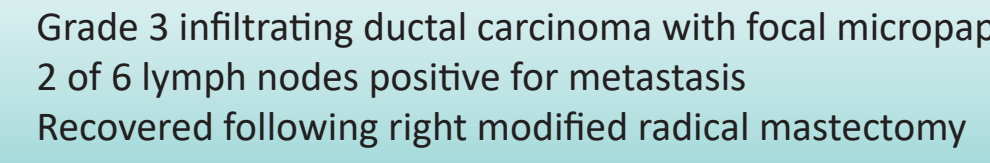

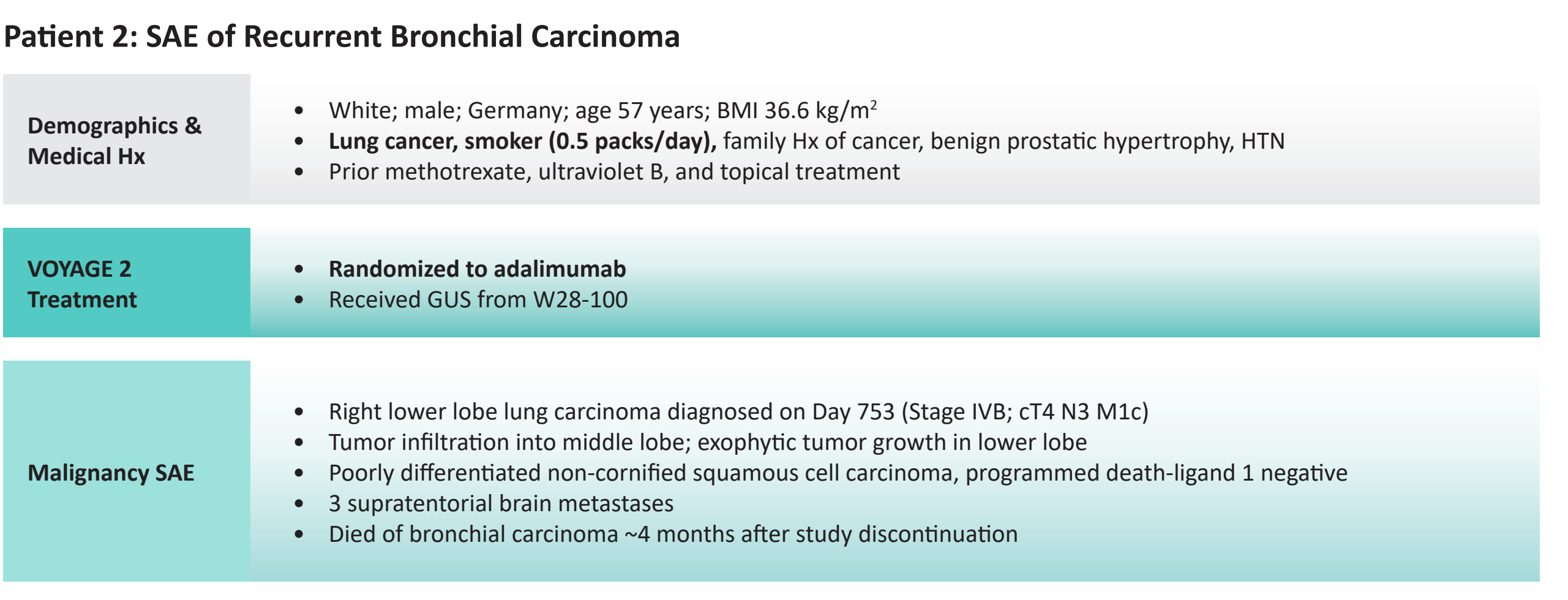

Patient 3: SAE of Invasive Melanoma

Demographics \&
Medical Hx

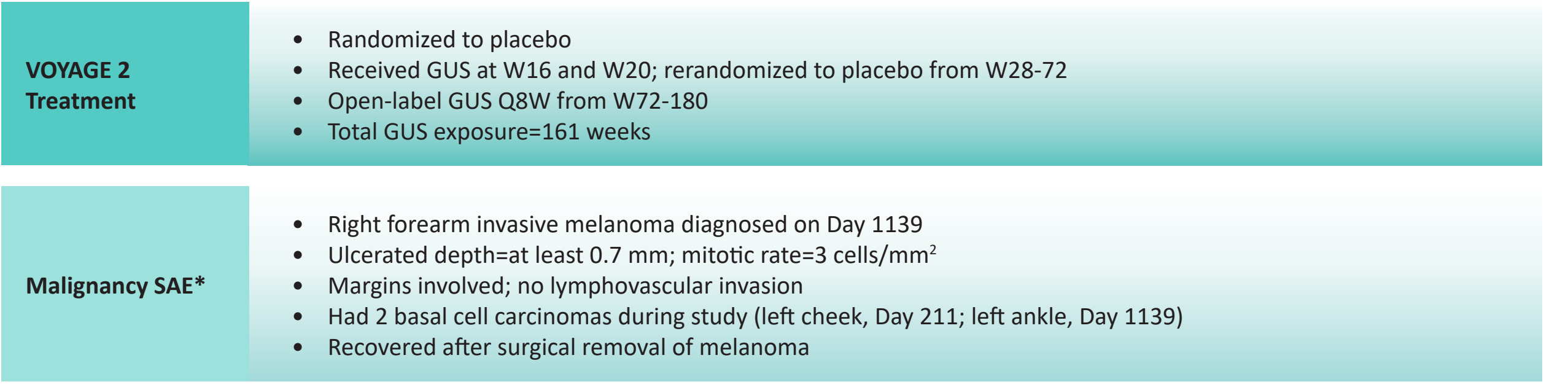

SAEs Other Than Malignancies

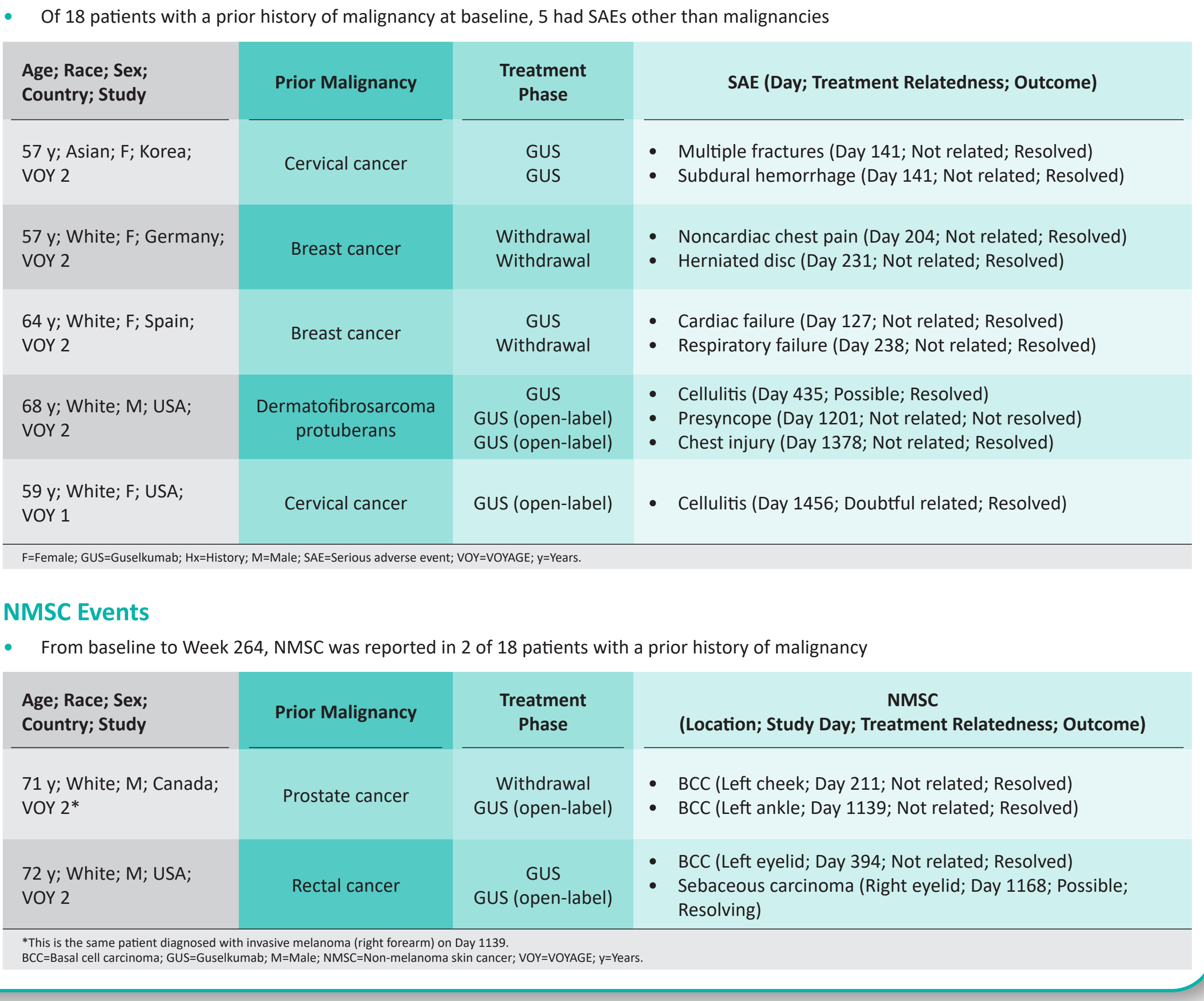

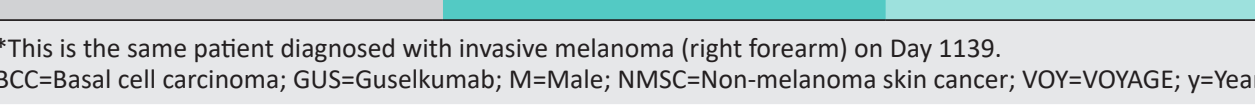

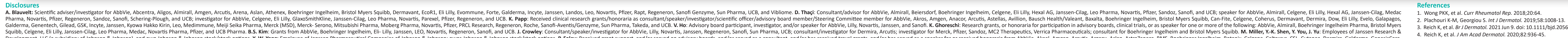

